\title{
Edlan-Mejchar Vestibular Deeping in a Failing Implant Case
}

\author{
Shabeer Mohamed Madani, Biju Thomas
}

\begin{abstract}
Necessity of the width of keratinized mucosa around implants has been a topic of much controversy. There have been reports both supporting and against the idea. This is a case report describing an EdlanMejchar vestibular deepening procedure done with respect to a mandibular anterior failing implant. The procedure helped in increasing the keratinized mucosa around the implant thus rendering a harmonious soft tissue for healing of the peri-implant mucosa. Based on the findings of the present case it can be concluded that keratinized mucosa around the implant is detrimental in the success of the implant.
\end{abstract}

Keywords: Edlan-Mejchar vestibuloplasty, Keratinized mucosa, Peri-implant soft tissue.

How to cite this article: Madani SM, Thomas B. Edlan-Mejchar Vestibular Deeping in a Failing Implant Case. Int J Oral Implantol Clin Res 2013;4(3):108-111.

Source of support: Nil

Conflict of interest: None declared

\section{INTRODUCTION}

Following the placement of an implant/abutment, the soft tissue healing around the implant may be composed of the lining (nonkeratinized) or the masticatory (keratinized) mucosa. The width of attached gingiva around natural teeth was considered important as it was necessary to dissipate the forces of the muscle pull of unattached mucosa and also reduces plaque retention. Also, since it was of keratinized origin it also was thought to be suitable to withstand tooth brushing and masticatory trauma. ${ }^{1}$ Lang and Loe in 1972 stated that a minimum of $2 \mathrm{~mm}$ of keratinized mucosa is required for adequate soft tissue health. ${ }^{2}$ Later in the early 1980s Wennstrom, Lindhe and Nyman in their studies proved that attached gingiva and its width have little role in the maintenance of periodontal health. ${ }^{3-5}$ But these same conclusions cannot be extrapolated to implants as the connective tissue fibers run parallel to the implant surface whereas these fibers are attached to the cementum in case of teeth. Thus there have been controversies over the necessity of adequate width of attached gingiva in case of implants. A recent systematic review by Carlos Brito et al in 2013 concluded that keratinized gingiva is indispensible to maintain peri-implant health. ${ }^{6}$

Edlan Mejchar procedure is a vestibular deepening procedure which was given by Edlan and Mejchar in 1963.7 It is a predictable technique with long-term results. This procedure also appeared to increase the width of the attached gingiva where other procedures were impracticable due to lack of vestibular depth.

This paper describes the case report of a patient in whom vestibular extension was carried out by the technique described by Edlan and Mejchar to correct a failing implant.

\section{CASE REPORT}

A male patient aged 27 years of age came to the department of periodontics with a failing implant with respect to 31 and exposed bone graft and membrane. On history patient revealed placement of an implant 4 months back with exposure of the implant 2 months postoperative for which a second stage surgery was done consisting of bone grafting and membrane placement. On examination the GTR membrane with particulate bone graft material and debris was exposed and slight mobility was noticed with respect to the implant at 31 . There was absence of attached gingiva at the site and vestibular depth was assessed to be $2 \mathrm{~mm}$. The treatment plan included first stage surgery which involved debridement of the area. After debridement the dehiscence was appreciated with respect to the buccal cortical plate and exposure of the implant was apparent (Fig. 1). The flap was sutured back to position by releasing the tension on the flap with the help of a horizontal incision (Fig. 2).

The second stage surgery involved the Edlan Mejchar vestibular deepening procedure which would help increase the vestibular depth and attached gingiva to relieve tension on the marginal gingiva and better adaptation of the soft tissue onto the implant for better peri-implant health.

Prior to the surgical procedure, the distance between the gingival margin and the depth of the vestibule was measured with the help of a William's graduated probe and was assessed to be $2 \mathrm{~mm}$ (Fig. 3). The frenal pull on the gingival margin can also be appreciated preoperatively (Fig. 4).

\section{Edlan-Mejchar Procedure}

First 2 vertical incisions placed mesial to the mandibular canines and starting at the mucogingival junction extending up to a distance of 10 to $12 \mathrm{~mm}$ on to the lower lip. The two vertical incisions were joined by a horizontal incision across the midline (Fig. 5). Then a split thickness flap was elevated separating the labial mucosa from the underlying muscular tissue using sharp dissection. This resulted in a loose flap of labial mucosa with its base on the gingiva (Fig. 6). 


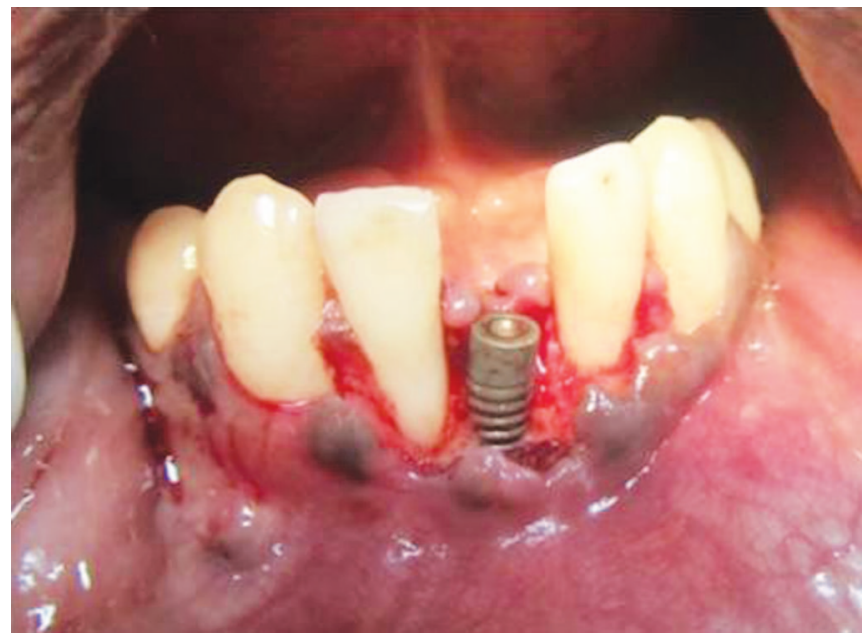

Fig. 1: Preoperative after debridement

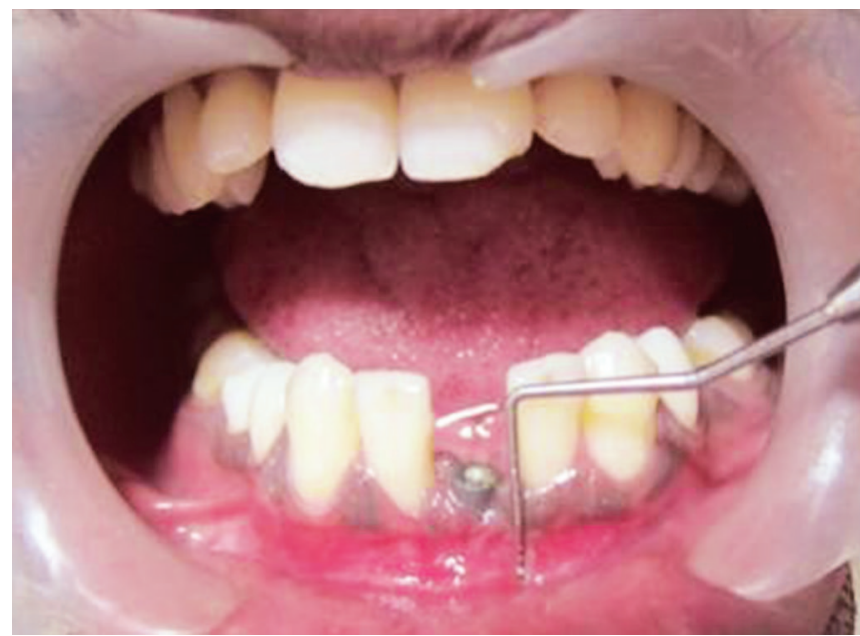

Fig. 3: Three weeks postoperative, soft tissue healing appreciated with $3 \mathrm{~mm}$ vestibular depth

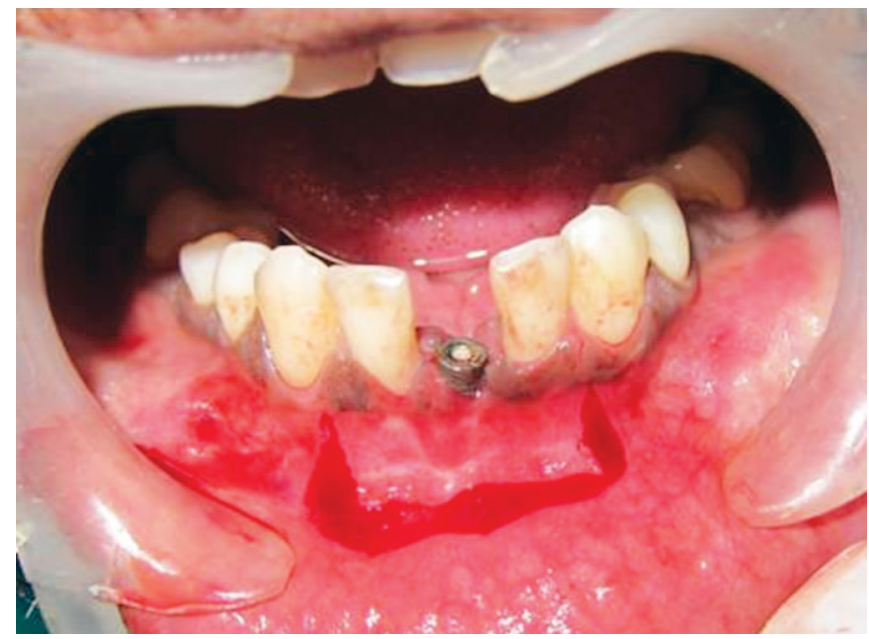

Fig. 5: The horizontal and vertical incisions placed

Incision and reflection of the periosteum: the loose flap of labial mucosa was folded upward and a horizontal incision was made on the periosteum extending between the 2 initial vertical incisions mesial to the canines. The periosteum was then separated from the bone with blunt dissection,

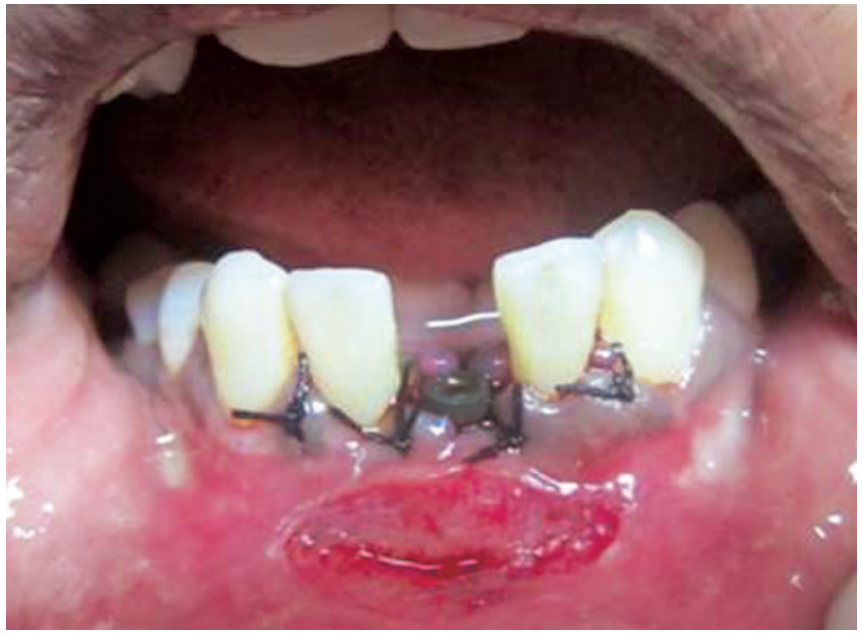

Fig. 2: 1st stage surgery: frenotomy and interrupted sutures placed

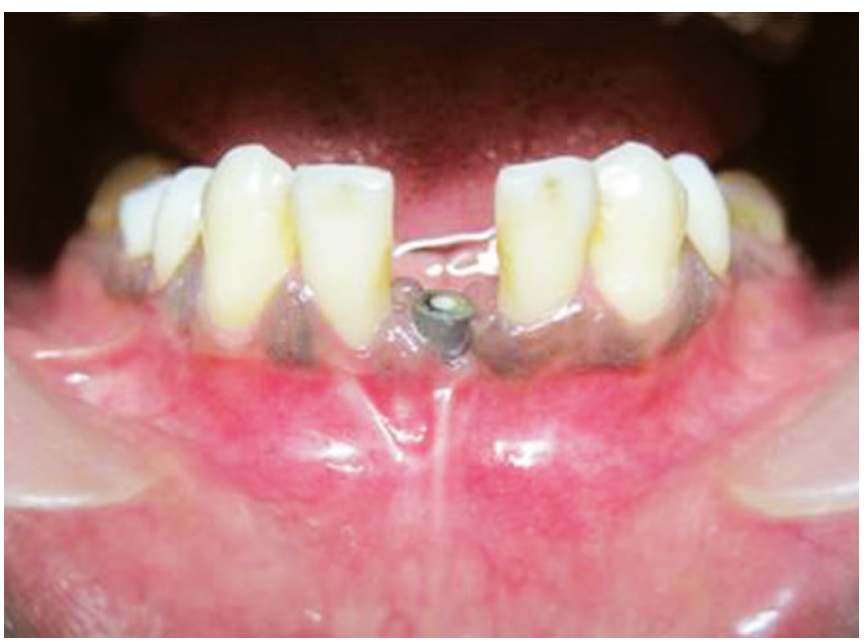

Fig. 4: Three weeks postoperative, can appreciate the frenal pull on the marginal gingiva

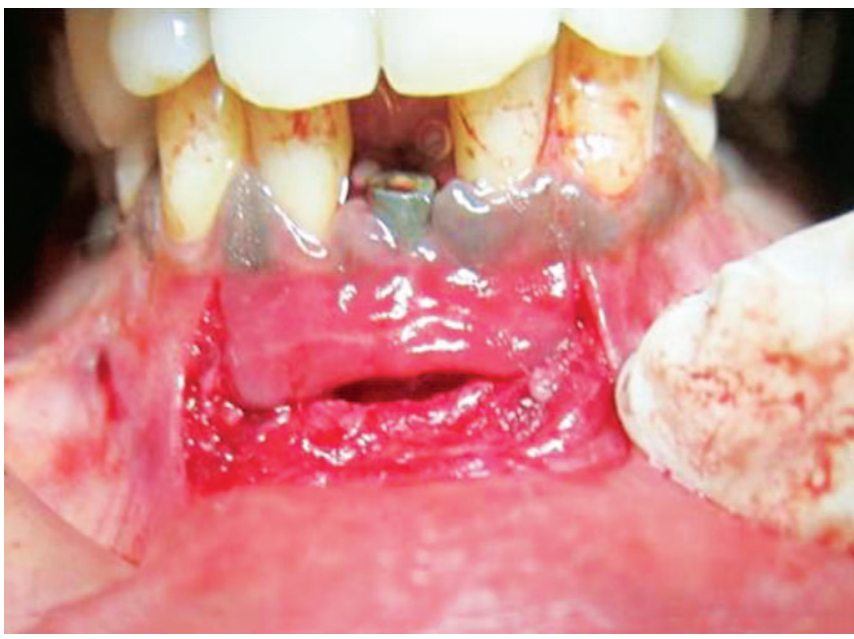

Fig. 6: Split thickness labial flap raised

forming a second flap with its base on the apical portion of the mandible (Fig. 7).

Transposition of the two flaps: the split thickness flap of labial mucosa was folded back and placed on the bone from which the periosteum had been removed. It was fixed with 


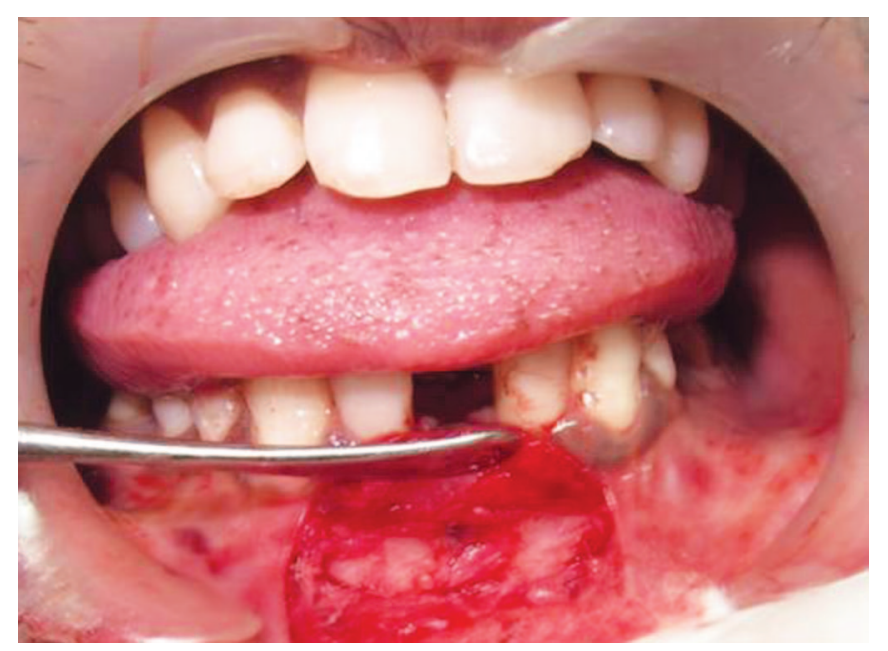

Fig. 7: Full thickness mucoperiosteal flap raised

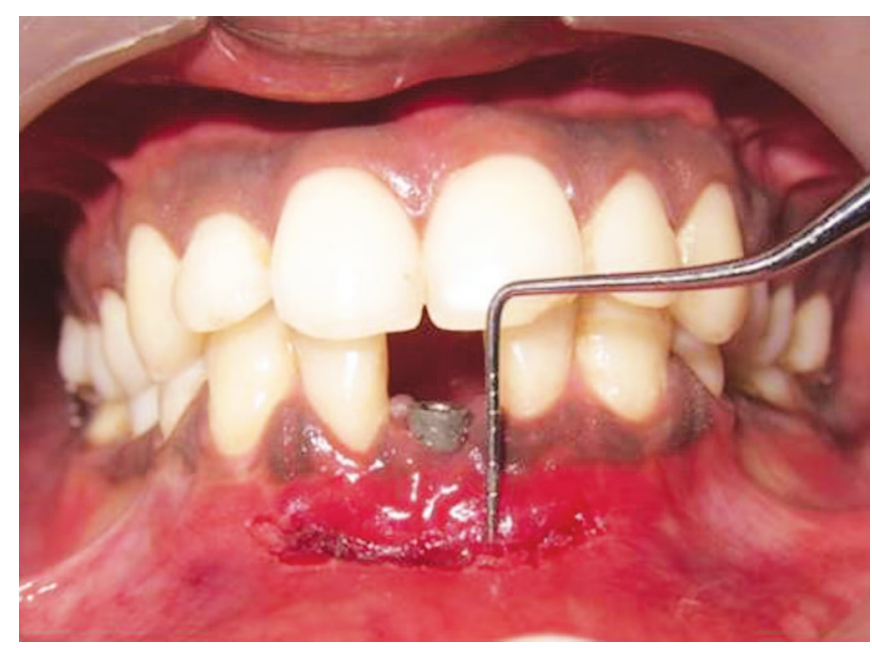

Fig. 9: Three weeks postoperative

interrupted sutures to the inner surface of the periosteum, which had been removed from the bone (Fig. 8).

A periodontal dressing was placed to protect the operated area. Amoxicillin $500 \mathrm{mg}$ TID for 5 days and anti-inflammatory (diclofenac $50 \mathrm{mg}$ ) BD for 5 days were prescribed to the patient in addition to chlorhexidine rinses. Other postsurgical instructions included intermittent cold fomentation on the first postoperative day, soft/liquid diet for 1 week, and maintenance of good oral hygiene. The patient recalled in 2 weeks time for review.

Postoperative recall: the 3 weeks postoperative examination revealed excellent healing (by first intention) and a considerable gain in the width of the attached gingiva and depth of the vestibule (up to $8 \mathrm{~mm}$ ) (Fig. 9). The patient was subsequently placed on a recall program and his periodontal condition was periodically reviewed. No loss of width of the attached gingiva was observed throughout the recall program and prosthetic phase was completed within 2 months time (Figs 10A and B).

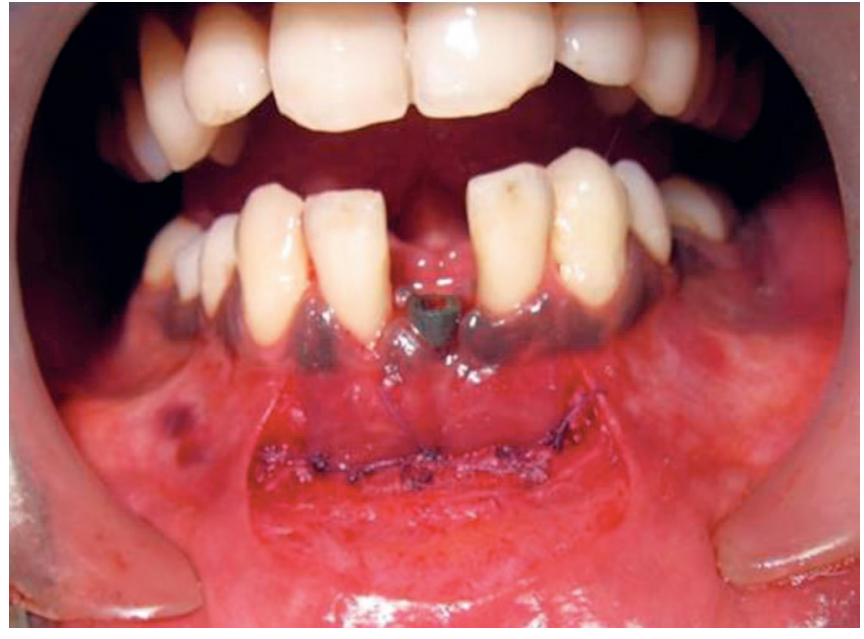

Fig. 8: Labial mucosa adapted and sutured onto the bone
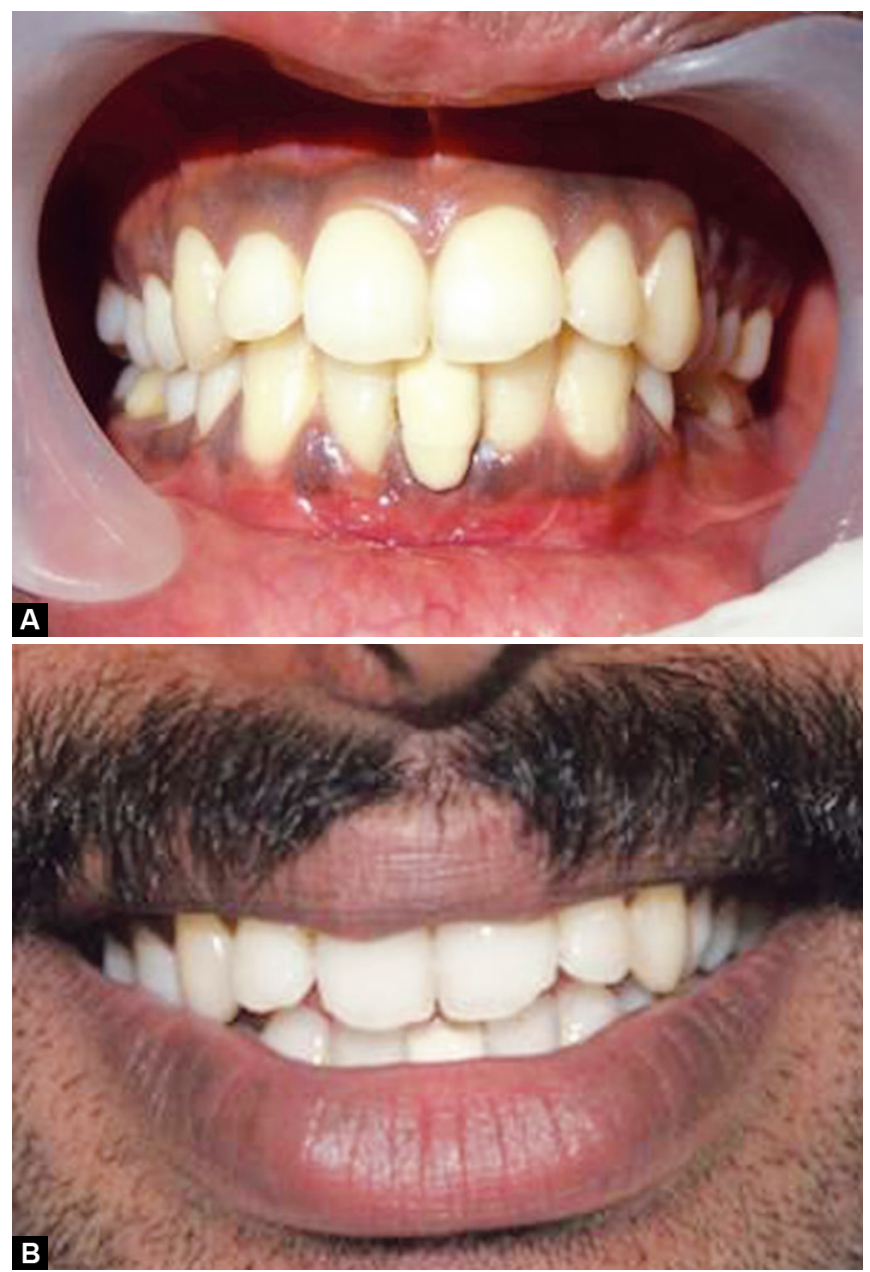

Figs 10A and B: Four months postoperative after prosthetic phase

\section{DISCUSSION}

The technique used here was a modification of the Kazanjian technique. The advantage of this technique is that the bone is not exposed and there is minimal contraction of the vestibular depth gained and scar formation is minimal. In this case there was an appreciable increase in the vestibular depth up to 
$7 \mathrm{~mm} 3$ weeks postoperative and the results were consistent 4 months postoperatively. A 5 years follow-up study done by Axel Ergenholtz and Anders Hugoson concluded that there was an average gain of $7.7 \mathrm{~mm}$ and insignificant loss of depth during the first 3 months which was followed by a gain during 5 years follow-up. ${ }^{8}$ Though there was an increase in the vestibular depth it is not without drawbacks. The major drawbacks which was observed is the patients complain of stiffness during movement of the lower lip which the patient got accustomed to within 1 month time. Literature also reports about a major drawback of the shortening of the lip, ${ }^{8}$ which was not seen in this case.

With regard to the peri-implant health, the tissues were normal with adequate amount of keratinized mucosa covering the implant. The mobility of the implant had improved. There was postoperative exposure of the implant which was masked with the help of the prosthetic crown.

Thus, based on the findings of the present case it can be concluded that keratinized mucosa around the implant is detrimental in the success of the implant. The technique advocated by Edlan and Mejchar can be used to increase the width of the keratinized mucosa around the implant predictably. Thus improving the overall prognosis.

\section{REFERENCES}

1. Mehta P, Peng LL. The width of attached gingiva- much ado about nothing? J Dent 2010;38:517-525.

2. Lang NP, Löe H. The relationship between the width of keratinized gingiva and gingival health. J Periodont 1972;43: 623-627.
3. Wennström J, Lindhe J. Plaque-induced gingival inflammation in the absence of attached gingiva in dogs. J Clin Periodont 1983;10:266-276.

4. Wennström J, Lindhe J. Role of attached gingiva for maintenance of periodontal health. Healing following excisional and grafting procedures in dogs. J Clin Periodont 1983;10:206-221.

5. Wennström J, Lindhe J, Nyman S. Role of keratinized gingiva for gingival health. Clinical and histologic study of normal and regenerated gingival tissue in dogs. J Clin Periodont 1981;8: 311-328.

6. Brito C, Tenenbaum HC, Wong BKC, Schmitt C. Nogueira-Filho $\mathrm{G}$. Is keratinized mucosa indispensable to maintain peri-implant health? A systematic review of the literature. J Biomed Mater Res Part B App Biomat 2013;00B:1-8.

7. Edlan A, Mejchar B. Plastic surgery of the vestibulum in periodontal therapy. Int Dent J 1963;13:593-596.

8. Bergenholtz, Axel, Hugoson A. Vestibular sulcus extension surgery in the mandibular front region: the Edlan-Mejchar Method-A 5-Year follow-up study. J Periodont 1973;14(5): 309-311.

\section{ABOUT THE AUTHORS}

\section{Shabeer Mohamed Madani (Corresponding Author)}

Postgraduate Student, Department of Periodontology, AB Shetty Memorial Institute of Dental Sciences, Deralakatte, Karnataka, India Phone: 08242204572, e-mail: shabeermohd_@hotmail.com

\section{Biju Thomas}

Professor and Head, Department of Periodontology, AB Shetty Memorial Institute of Dental Sciences, Deralakatte, Karnataka, India 